\title{
Cyclin D1 overexpression and p53 inactivation immortalize primary oral keratinocytes by a telomerase-independent mechanism
}

\author{
Oliver G. Opitz, ${ }^{1,2,3}$ Yasir Suliman, ${ }^{1,2}$ William C. Hahn,,${ }^{4,5}$ Hideki Harada,, 2 \\ Hubert E. Blum, ${ }^{3}$ and Anil K. Rustgi $1,2,6,7$ \\ ${ }^{1}$ Division of Gastroenterology, and \\ ${ }^{2}$ Abramson Family Cancer Research Institute, University of Pennsylvania, Philadelphia, Pennsylvania, USA \\ ${ }^{3}$ Department of Medicine, University of Freiburg, Freiburg, Germany \\ ${ }^{4}$ Whitehead Institute for Biomedical Research, Cambridge, Massachusetts, USA \\ ${ }^{5}$ Department of Adult Oncology, Dana-Farber Cancer Institute, and Department of Medicine, \\ Brigham and Women's Hospital, Boston, Massachusetts, USA \\ ${ }^{6}$ Cancer Center, and \\ ${ }^{7}$ Department of Genetics, University of Pennsylvania, Philadelphia, Pennsylvania, USA
}

Address correspondence to: Anil K. Rustgi, 600A CRB, Division of Gastroenterology, University of Pennsylvania, 415 Curie Boulevard, 600a CRB, Philadelphia, Pennsylvania 19104-6144, USA.

Phone: (215) 898-0154; Fax: (215) 573-5412; E-mail: anil2@mail.med.upenn.edu.

Received for publication December 4, 2000, and accepted in revised form June 28, 2001.

The immortalization of human cells is a critical step in multistep carcinogenesis. Oral-esophageal carcinomas, a model system to investigate molecular mechanisms underlying squamous carcinogenesis, frequently involve cyclin D1 overexpression and inactivation of the p53 tumor suppressor. Therefore, our goal was to establish the functional role of cyclin D1 overexpression and p53 inactivation in the immortalization of primary human oral squamous epithelial cells (keratinocytes) as an important step toward malignant transformation. Cyclin D1 overexpression alone was found to induce extension of the replicative life span of normal oral keratinocytes, whereas the combination of cyclin D1 overexpression and p53 inactivation led to their immortalization. This study also demonstrates that immortalization of oral keratinocytes can be independent of telomerase activation, involving an alternative pathway of telomere maintenance (ALT).

J. Clin. Invest. 108:725-732 (2001). DOI:10.1172/JCI200111909.

\section{Introduction}

Oral-esophageal squamous epithelial cells share major structural and functional features with the well-characterized skin keratinocyte systems and provide a good model to study basic keratinocyte biology as well as processes of immortalization and malignant transformation. Keratinocytes undergo an exquisite program of proliferation and differentiation in vivo. Insights into underlying molecular mechanisms in oralesophageal keratinocytes have been gained through in vitro and in vivo models $(1,2)$.

Normal or primary keratinocytes display a restricted replicative life span in cell culture. Those cells initially proliferate but eventually enter a state of permanent growth arrest, called replicative senescence (3), which is clearly distinct from terminal differentiation. Senescence is accompanied by several genetic changes including an increase in expression of several inhibitors of cyclin-dependent kinases (cdk's), as well as telomere shortening $(4,5)$. It has been suggested that senescence forms a barrier against tumorigenesis and that acquisition of the ability to proliferate an unlimited number of times, termed immortalization, is an essential step in the malignant transformation of normal cells (6). Tumor cells escape this growth control checkpoint and therefore can provide some insight into the mechanisms involved in the pathway from senescence to immortality. Furthermore, some of the most common known genetic changes in cancer development, such as the inactivation of the $\mathrm{p} 53$ and $\mathrm{pRb}$ pathways, play a critical role in cellular immortalization (7). As a separate consideration, cell culture conditions may contribute to immortalization (8).

In addition to changes in oncogenes and tumor suppressor genes, the gradual loss of DNA from the ends of telomeres has been implicated in the control of the proliferative potential of cells (9). Shortened telomeres at a critical length are thought to provide the signal to activate the program of cellular senescence. Telomerase is an enzyme that restores telomeric DNA sequences, and expression of its activity is thought to be involved in immortalization of human cells in vitro and eventually tumor progression in vivo (10). Telomerase activity has been detected in the majority of different tumor types, including oral squamous cell carcinoma (11). Presenescent human cells lack telomerase, and ectopic expression 
of hTERT (the catalytic subunit of telomerase) in presenescent human fibroblasts or retinal pigment epithelial cells was found to immortalize these cell types (12). By contrast, it has been shown that ectopic expression of hTERT is not sufficient to immortalize normal human epithelial cells and that additional loss of the $\mathrm{pRb} / \mathrm{p} 16$ (INK4a) cell cycle control is required $(13,14)$. Generally, one can also bypass senescence through inactivation of the $\mathrm{pRb}$ and $\mathrm{p} 53$ tumor suppressor pathways, e.g., by SV 40 large T antigen (15). Such cells remain telomerase negative and eventually enter crisis characterized by cell death. Activation of telomerase will permit such cells with critically short telomeres to become immortal. Therefore, the most common method to generate immortalized cells is with oncogenic viruses such as SV 40 or human papillomavirus (HPV) $(16,17)$. Recently, it has been demonstrated that telomerase can cooperate with SV $40 \mathrm{~T}$ antigen and ras to induce malignant transformation in normal human cells $(6,18)$.

Overexpression of the cyclin D1 oncogene is associated with human cancer and is the most common genetic alteration in human oral-esophageal squamous cell carcinomas $(19,20)$. Furthermore, cyclin D1 induces dysplasia in the oral-esophageal epithelia of transgenic mice (21). Cyclin D1 associates with cdk 4 and 6, and the complex phosphorylates and inactivates $\mathrm{pRb}$; inactivating mutations of $\mathrm{pRb}$ are not observed in oral-esophageal squamous cell carcinomas. By contrast, p53 mutations are frequently found in oral-esophageal cancer. Furthermore, $\mathrm{p} 53$ is inactivated in a high proportion of oral dysplastic lesions, implicating a role in the initiation of immortalization in this cell type (22). Following these patterns of genetic events seen during in vivo tumor development, we sought to identify the role of cyclin D1 overexpression and functional p53 inactivation in the immortalization of human oral squamous epithelial cells or keratinocytes. Cyclin D1 alone or in combination with dominant-negative p53 was ectopically overexpressed in normal human oral keratinocytes in culture using retroviral transduction. Whereas cyclin D1 overexpression extended the replicative life span of primary human oral keratinocytes, the combination with dominant-negative p53 resulted in immortalization of these cells. Importantly, immortalization under these conditions was independent of telomerase activity.

\section{Methods}

Cell culture and retroviral infection. Normal diploid human oral keratinocytes (OKF6) were established from a biopsy of normal floor of the mouth of clinically and genetically normal tissue. OKF6 cells have been cryopreserved within their first two serial passages in culture and characterized extensively (23). OKF6 and the lines generated thereof (OKF6-LacZ, OKF6-D1, OKF6- $\Delta \mathrm{p} 53$, OKF6-D $1 / \Delta \mathrm{p} 53$ ) were grown in defined keratinocyte-SFM with defined growth supplement (Life Technologies Inc., Rockville, Maryland, USA) and final $\mathrm{Ca}^{2+}$-concentration of $0.4 \mathrm{mM}$. The medium was supplemented with antibiotics. To assess mitogen dependence, cells were plated in defined keratinocyteSFM supplemented with a reduced concentration $(10 \%)$ of growth factors. The amphotropic packaging cell line Phoenix A was grown in supplemented DMEM (Sigma Chemical Co., St. Louis, Missouri, USA) and then transiently transfected with the respective retroviral vector to generate amphotropic retroviruses. Retroviral supernatant was harvested 48 hours after transfection and filtered through a $0.45-\mu \mathrm{m}$ filter, and fresh retroviral supernatant was used for infection of exponentially growing OKF6 cells. Medium containing puromycin $(1 \mu \mathrm{g} / \mathrm{ml})$ and/or G418 $(400 \mathrm{mg} / \mathrm{ml})$ was exchanged to start selection 48 hours after infection. In each set of experiments, multiple clones were pooled for further processing after 1-2 days of puromycin selection and/or 5 days of G418 selection. The retroviral expression vectors PBPSTR-D1 and PBABE-LacZ were obtained from S.A. Reeves (Massachusetts General Hospital, Boston, Massachusetts, USA) (24). The inducible retroviral vector PBPSTR-D1 contains both elements of the tetracycline-regulated system and has been described previously by us and others (25). The gene of interest is expressed in the absence of tetracycline (TET-OFF vector system). The puromycin resistance gene under the control of the promoter within the $5^{\prime}$-LTR is present in both retroviral vectors. The LXSN vector containing the p53 coding sequence with a V143A mutation was used to generate a dominant-negative version of $\mathrm{p} 53$ (26).

Determination of replicative life span. Serial cultures of the different OKF6 lines (OKF6, OKF6-LacZ, OKF6D1, OKF6- $\Delta \mathrm{p} 53$, OKF6-D $1 / \Delta \mathrm{p} 53)$ were performed in $10-\mathrm{cm}$ dishes by plating $10^{5}$ cells, refeeding the cells every 2 nd day, and subculturing every $4-5$ days. The doubling number of each passage was calculated using the formula $\mathrm{PD}=(\mathrm{nf} / \mathrm{n} 0) / \log 2$, where $\mathrm{n} 0$ is the initial number of cells and $\mathrm{nf}$ is the final number of cells. Cellular immortalization was defined as cell growth of at least three times beyond the life span of the parental cells.

Flow cytometry. Exponentially growing cells were collected at different population doubling (PD) times and centrifuged at $300 \mathrm{~g}$ for 4 minutes. The cell pellet was resuspended in $0.5 \mathrm{ml}$ of PBS, fixed in $5 \mathrm{ml} 70 \%$ ethanol, and stored at $-20^{\circ} \mathrm{C}$ overnight. The cells were then washed twice with PBS and resuspended in a 1-ml solution containing $3.8 \mathrm{mM}$ sodium citrate and $10 \mu \mathrm{g} / \mathrm{ml}$ propidium iodide. After $10 \mathrm{mg} / \mathrm{ml}$ of RNase treatment at $37^{\circ} \mathrm{C}$ for 20 minutes, the samples were analyzed by a fluorescence-activated cell sorter (FACScan; Becton Dickinson \& Co., Franklin Lakes, New Jersey, USA).

Western blot analysis. Lysates from exponentially growing cells were harvested in a buffer $(50 \mathrm{mM}$ HEPES [pH 7.4], $0.1 \%$ Nonidet P-40, and $250 \mathrm{mM} \mathrm{NaCl}$ ) with $1 \mathrm{mM}$ protease and $10 \mathrm{mM}$ phosphatase inhibitors. A total of $10 \mu \mathrm{g}$ of total protein was separated on $10 \%$ SDS-polyacrylamide gels and transferred to Immobilon membranes (Millipore Corp., Bedford, Massachusetts, USA). Blocking was performed in 5\% milk, 10 

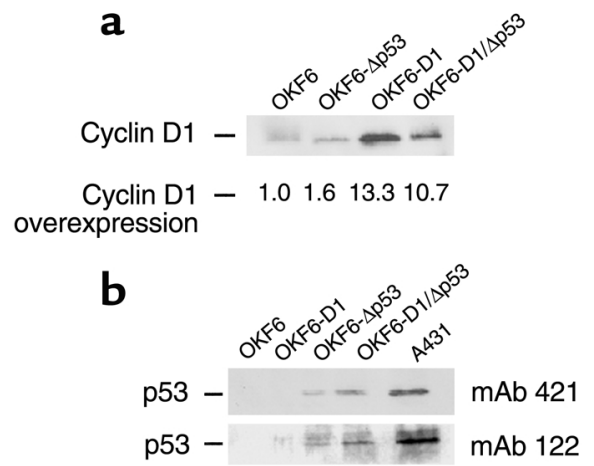

\section{Figure 1}

Effects of cyclin D1 overexpression and/or dominant-negative p53 expression in oral keratinocytes (OKF6). (a) Equal amounts of protein for parental OKF6, OKF6- $\Delta$ p53, OKF6D1, and OKF6-D1/ $\Delta$ p53 were separated on a 10\% SDS-PAGE. The level of cyclin D1 overexpression was compared by subsequent immunoblot analysis using polyclonal cyclin D1 antibody (H295) and monoclonal cyclin D1 antibody (HD11) (data not shown). (b) Equal amounts of protein for parental OKF6, OKF6-D1, OKF6- $\Delta$ p53, and OKF6-D1/ $\Delta$ p 53 were separated on a $10 \%$ SDS-PAGE. Subsequent immunoblot was done with two different conformational p53 antibodies: the monoclonal p53 antibody 421 and the monoclonal p53 antibody 122 . The human epidermoid cancer cells A431 served as positive control.
$\mathrm{mM}$ Tris-HCl (pH 7.4), $150 \mathrm{mM} \mathrm{NaCl}$, and $0.2 \%$ Tween-20 for 1 hour, followed by incubation with primary antibodies as indicated $(1: 3,000)$. The secondary antibody was either peroxidase-conjugated anti-mouse or anti-rabbit Ig (1:2,500; Amersham Corp., Burlington, Massachusetts, USA). Detection was by chemiluminescence (ECL; Amersham Corp.). Primary antibodies used were monoclonal cyclin D1 antibody (HD11), polyclonal cyclin D1 antibody (H295), monoclonal p53 antibody 421, monoclonal p53 antibody 122 , and monoclonal p16 antibody (JC8). Quantification was done using Image software (National Institutes of Health, Bethesda, Maryland, USA).

TRAP assays/telomeric-length assays. Cellular extracts of all generated oral keratinocytes were assayed for telomerase activity using the PCR-based telomerase repeat amplification protocol (TRAP) assay (27). Cellular extracts (50 and $500 \mathrm{ng}$ ), along with a heat-inactivated controls, were used for TRAP assays. Telomere length was measured by hybridizing a ${ }^{32} \mathrm{P}$-labeled telomeric (CCCTAA) probe to $10 \mu \mathrm{g}$ of Hinfl- and RsaI-digested genomic DNA as described previously (27).

Karyotypic analysis. Exponentially growing cultured OKF6 and OKF6-D1/ $\Delta$ p53 cells were incubated with $10^{-7} \mathrm{M}$ Colcemid (Life Technologies Inc.) for 1 hour, resuspended in warm hypotonic buffer $(0.075 \mathrm{M} \mathrm{KCl})$, allowed to swell for $10-15$ minutes at $37^{\circ} \mathrm{C}$, fixed by addition of cold ethanol/acetic acid, and applied to chilled microscope slides. Chromosome preparations of cultured cells were counted for the number of metaphase spreads. Metaphase spreads (> 10) were then analyzed at different PD times at the University of Pennsylvania Cytogenetics Core Facility.

Subcutaneous tumorigenicity assays. Six- to eight-week-old immunocompromised athymic nude mice (NIH III; Charles River Laboratories, Wilmington, Massachusetts, USA) were injected with a cell suspension containing $2 \times 10^{6}$ exponentially growing cultured OKF6, OKF6$\mathrm{D} 1 / \Delta \mathrm{p} 53$, SCC15 (oral squamous cancer cell line), or TE 12 (esophageal squamous cancer cell line) cells. Tumor size was measured every 3 days. The time of initial tumor formation was defined as the time when the tumor had reached a diameter of $3 \mathrm{~mm}$. Mice were sacrificed when the tumors grew to $1 \mathrm{~cm}$ in diameter or after 12 weeks of monitoring and were subsequently analyzed.

\section{Results}

Ectopic cyclin D1 overexpression in normal human oral keratinocytes induces cell-cycle abnormalities and extension of replicative life span. To elucidate the role of the cyclin D1 oncogene in the immortalization of oral squamous epithelial cells, we ectopically overexpressed cyclin D1 in normal human oral keratinocytes. To overexpress cyclin D1, we used a tetracycline-regulated retroviral vector system, pBPSTR-D1, containing the human cyclin D1 cDNA, to infect the normal human oral keratinocytes (OKF6). OKF6 cells have been described previously and shown not to bear any genetic alterations $(14,23)$.

A tetracycline-inducible system was used for infection in order to prevent any potential toxic effects of cyclin D1 overexpression. Given that no toxicity was observed,

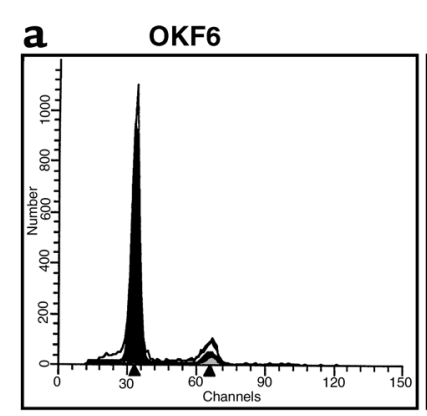

G1: $83 \% ; \mathrm{S}: 8 \% ; \mathrm{G} 2 / \mathrm{M}: 9 \%$

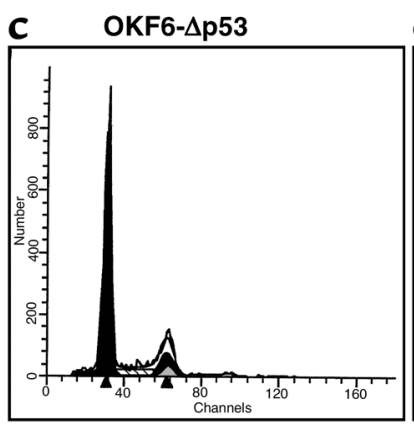

G1: $70 \%$; S: $14 \% ;$ G2/M: $15 \%$

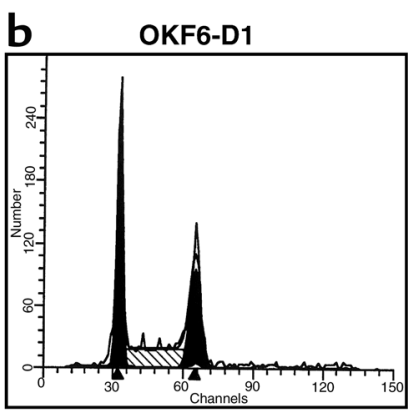

G1: $40 \%$; S: 31\%; G2/M: 30\%

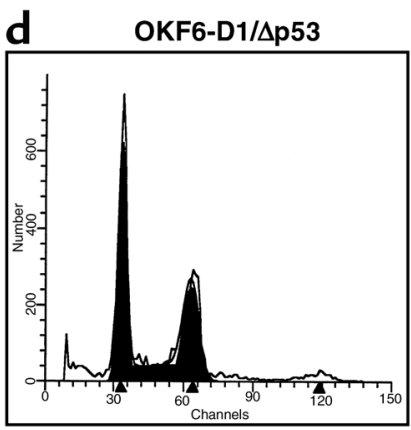

G1: $52 \% ;$ S: $27 \%$; G2/M: $20 \%$

\section{Figure 2}

Cell-cycle pattern of the parental and derived oral keratinocytes. Cellcycle analysis of OKF6, OKF6-D1, OKF6- $\Delta$ p53, and OKF6-D1/ $\Delta \mathrm{p} 53$ cells was done under standard conditions using FACScan. Cell-cycle analysis is displayed either as a histogram or as a table. (a) OKF6; (b) OKF6-D1; (c) OKF6- $\Delta$ p53; and (d) OKF6-D1/ $\Delta$ p53. 


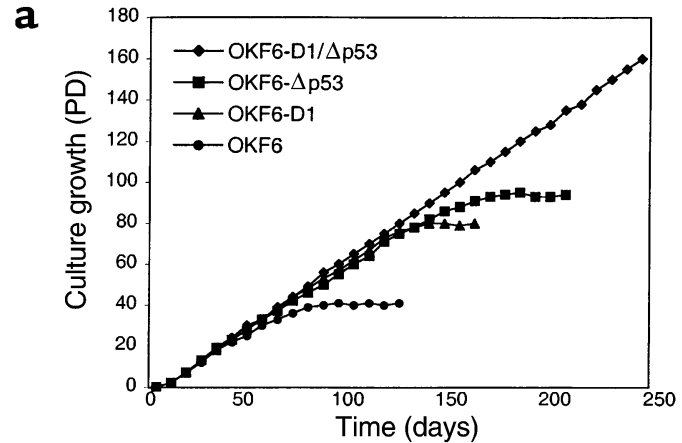

\begin{tabular}{lll}
\hline Cell line & Replicative life span \\
\hline OKF6 & 40 PDs & \\
OKF6-lacZ & 40 PDs & \\
OKF6-D1 & 80 PDs & extended \\
OKF6- $\Delta$ p53 & 100 PDs & extended \\
OKF6-D1/ $\Delta$ p53 & >160 PDs & immortal \\
\hline
\end{tabular}

\section{Figure 3}

Replicative life span of the parental and derived oral keratinocytes. (a) The growth characteristics of the parental and derived OKF6 cells are depicted as follows: OKF6 (circles), OKF6-D1 (triangles), OKF6$\triangle \mathrm{p} 53$ (squares), and OKF6-D1/ $\Delta \mathrm{p} 53$ (diamonds). (b) The replicative life span of OKF6, OKF6-lacZ, OKF6-D1, OKF6- $\Delta$ p53, and OKF6-D1/ $\Delta p 53$ was assessed by calculating the PDs of each cell line. Immortalization was assessed if cells grew at least three times beyond the life span of the parental cells.

cyclin D1 was constitutively expressed (TET off) in the ensuing experiments (25). Infection efficiency was monitored and confirmed with a lacZ gene containing retrovirus (pBabe-lacZ). Infected OKF6 cells were selected with the appropriate antibiotic, and the selected clones were pooled to ensure polyclonality. Cells used for further studies were designated OKF6-D1. The levels of endogenous and ectopically expressed cyclin D1 in parental OKF6 and cyclin D1-infected cells (OKF6-D1 cells) were determined using Western blot analysis (Figure 1a).

Because cyclin D1 plays an important role in the G1phase progression of the cell cycle, we investigated the effects of cyclin D1 overexpression on cell-cycle dynamics in these cells. Flow cytometry analysis revealed pronounced cell-cycle redistribution in OKF6-D1 cells, with a $20 \%$ increase in cells cycling in S-phase compared with parental OKF6 cells (Figure 2, a and b). Similar results were observed in five separate cyclin D1 transduction experiments (data not shown).

\footnotetext{
Figure 4

Telomerase activity in the parental and derived oral keratinocytes. Cellular extracts ( $500 \mathrm{ng}$ and $50 \mathrm{ng}$ ) of OKF6, OKF6-lacZ, OKF6-D1, OKF6- $\Delta$ p53, and OKF6-D1/ $\Delta$ p53 cells were assayed for telomerase activity using the PCRbased TRAP-assay. Heat-treated (HT) samples served as negative control, and OKF6 cells transduced to express hTERT (13) were used as positive control. IC is an internal PCR-control to demonstrate the absence of PCR inhibitors in the cellular extracts.
}

We next investigated the growth of these cells on extended passage in culture. Both OKF6 cells and OKF6 cells transduced with the control vector (OKF6-LacZ) senesced at $40 \mathrm{PDs}$, as evidenced by growth arrest and enlargement of cells in culture (Figure 3). By contrast, the replicative life span of OKF6-D1 cells was significantly and reproducibly extended to $80 \mathrm{PDs}$, at which time the OKF6-D1 cells eventually senesced (Figure 3 ). Of note, the p16 tumor suppressor protein product was downregulated in early passages, but expression was high in presenescent passages of OKF6-D1 cells (data not shown).

Ectopic expression of dominant-negative $p 53$ in OKF6-D1 cells results in immortalization. Given that inactivation of p53 appears to be important in the early phases of oralesophageal carcinogenesis, we investigated the functional consequences of p53 inactivation in combination with cyclin D1 overexpression in human oral keratinocytes. Independent transduction experiments were done to express a dominant-negative version of the tumor suppressor gene p53 (V143A) in OKF6-D1 cells. Clones from each experiment were pooled and established as OKF6-D1/ $\Delta \mathrm{p} 53$ oral keratinocytes. To exclude the possibility that changes occurred as a consequence of specific genetic selection in one generated cell strain, a second strain from a different transduction experiment was established. Both strains revealed identical genetic features and growth properties.

The effect of the dominant-negative p53 mutant was demonstrated by stabilization of the p53 protein in infected cells as assayed by Western blot analysis using two different conformational p53 antibodies (Figure 1b). Both strains of OKF6-D1/ $\Delta \mathrm{p} 53$ cells revealed stabilized p53 protein as did OKF6- $\Delta$ p53 cells compared with OKF6-D1 cells or the parental oral keratinocytes, where no p53 signal was detected (Figure 1b). FACS analysis of OKF6-D $1 / \Delta \mathrm{p} 53$ cells revealed a cell-cycle redistribution comparable to that of the OKF6-D1 cells, whereas the OKF6- $\Delta$ p53 cells showed no significant cell-cycle redistribution (Figure 2, $\mathrm{c}$ and d). The FACS analysis was done at different PD times (early PD and late PD), but no major differences were observed (data not shown).

The OKF6-D1/ $\Delta \mathrm{p} 53$ cell strains revealed an extension of their replicative life span to more than $160 \mathrm{PDs}$ and

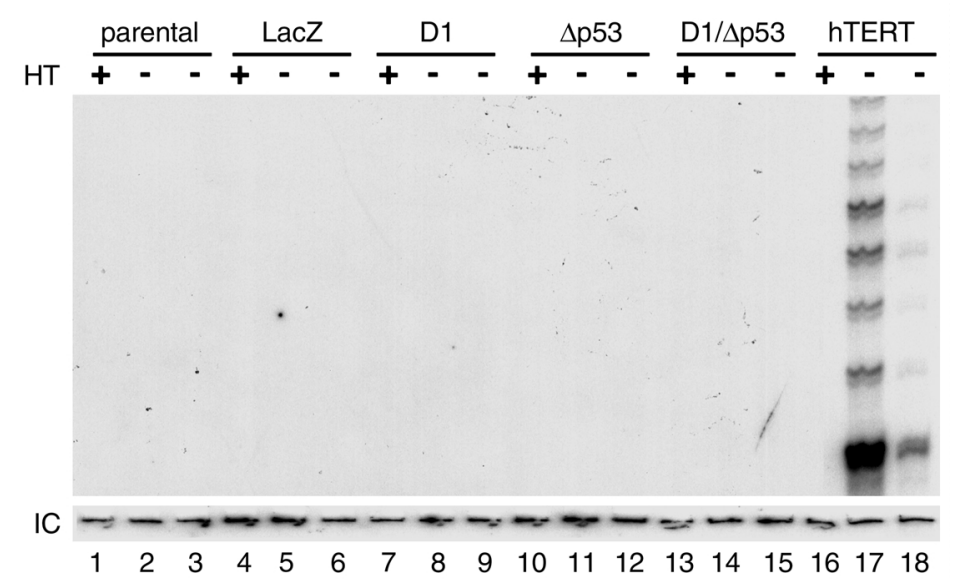


a

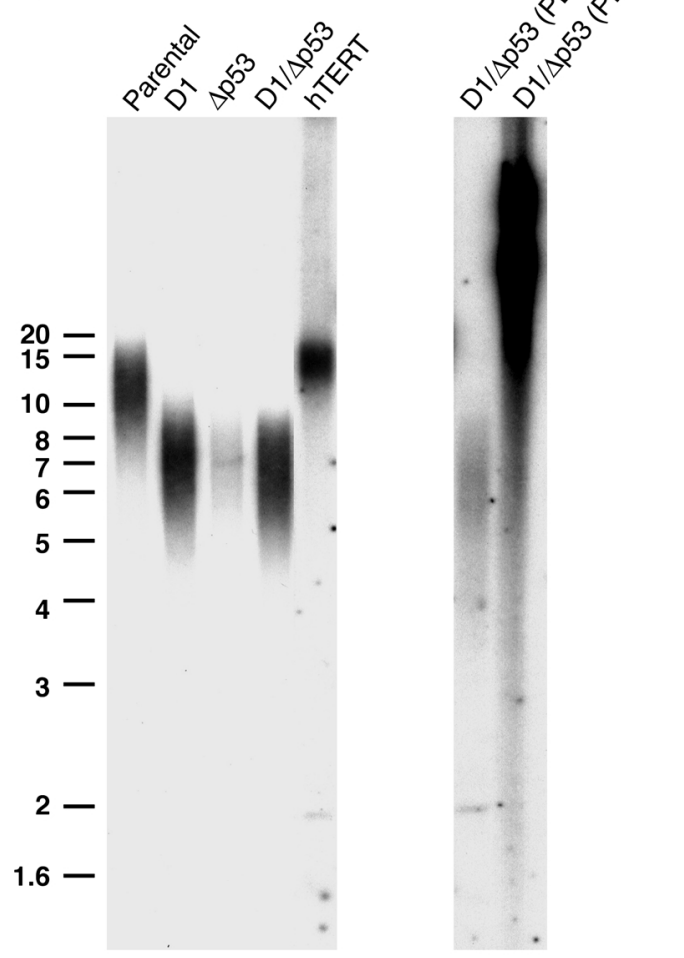

Figure 5

Telomere length in the parental and derived oral keratinocytes. (a) Telomere length for OKF6, OKF6-D1, OKF6- $\Delta$ p53, and OKF6-D1/ $\Delta \mathrm{p} 53$ (120 PDs) cells was analyzed by hybridization of genomic DNA with a specific oligonucleotide probe. OKF6-hTERT cells served as control. (b)

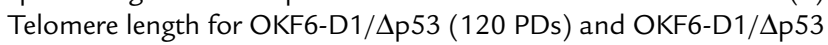
(160 PDs) cells. Size standard is indicated at the left. The broad smear in OKF6-D1/ $\Delta$ p53 (160 PDs) starts at a telomere length of $40 \mathrm{~kb}$.

continue to grow in culture (Figure 3). Thus, overexpression of both cyclin D1 and dominant-negative p53 reproducibly immortalizes human oral keratinocytes. It is conceivable that indefinite passage in culture will eventually result in the acquisition of other genetic alterations and frank transformation. We did not detect the onset of any widespread cell death while passaging the OKF6-D1/ $\Delta \mathrm{p} 53$ cell strains as observed during crisis in SV 40 immortalized cells. Apoptosis was not observed by light microscopic evaluation or by DNA-laddering (Figure 3 and data not shown) in spite of a small sub-G1 peak in the FACS analysis. Thus, the sub-G1 peak observed in the OKF6-D $1 / \Delta \mathrm{p} 53$ cells does not appear to be consistent with apoptosis.

OKF6- $\Delta$ p53 cells showed an extension of the replicative life span (100 PDs), which was slightly more pronounced than that in the OKF6-D1 cells, but they ultimately senesced (Figure 3). Interestingly, there was only a minimal increase in the polyploid cell population in OKF6- $\Delta$ p53 cells during serial passage (data not shown). Although p16 was downregulated in early-passage OKF6-D1 cells and then expressed in presenescent cells, it was continuously expressed in OKF6-D1/ $\Delta \mathrm{p} 53$ cells (data not shown).
Mechanism of immortalization in OKF6-D1/ $\Delta p 53$ cells. Immortal human cells maintain their telomeres by one of two known mechanisms: activation of telomerase or activation of an alternative pathway in lengthening telomeres (ALT).

To further investigate the mechanisms of immortalization in oral keratinocytes, we assayed the parental OKF6 cells and derivatives for telomerase activity. Telomerase activity was absent in all OKF6 cells (OKF6, OKF6-LacZ, OKF6-D1, OKF6- $\Delta$ p53, OKF6-D1/ $\Delta \mathrm{p} 53$ ), including the immortal OKF6-D1/ $\Delta \mathrm{p} 53$ cell strains (Figure 4). Telomere length measurements by Southern blotting with a probe specific for mammalian telomeric repeats (TRF) revealed a progressive shortening of telomeres that correlated with the replicative life-span extension of oral keratinocytes. Parental OKF6 cells showed a longer average telomere length than did OKF6-D1 and OKF6- $\Delta \mathrm{p} 53$ cells, which retained longer telomeres than OKF6-D1/ $\Delta \mathrm{p} 53$ cells at $120 \mathrm{PDs}$ (Figure 5). Interestingly, the OKF6-D1/ $\Delta \mathrm{p} 53$ cells at $160 \mathrm{PDs}$ revealed an elongation of telomere length as measured by TRF with a heterogeneous lengthening including very long telomeres of up to $40 \mathrm{~kb}$ (Figure 5). Thus, the OKF6-D1/ $\Delta \mathrm{p} 53$ cell strains acquired the ability to maintain their telomeres in the absence of telomerase activity at PD 120-160.

To assess for chromosomal recombination events in the OKF6-D1/Ap53 cells, karyotypic analysis was performed at different PD times. This revealed aneuploidy and consistent chromosomal rearrangements compatible with the selection of chromosomal changes in cells with critically short telomeres (Table 1 ).

Growth characteristics of immortalized OKF6-D1/ $\Delta p 53$ cells. Next, we determined whether the process of immortalization in OKF6-D1/ $\Delta \mathrm{p} 53$ cells impaired specific keratinocyte growth control mechanisms. We investigated the ability of OKF6, OKF6-D1, OKF6- $\Delta \mathrm{p} 53$, and OKF6$\mathrm{D} 1 / \Delta \mathrm{p} 53$ cells to grow in growth factor-reduced medi-

\section{Table 1}

Karyotype in oral keratinocytes

$\begin{array}{ll}\text { Cells } & \text { Karyotype } \\ \text { OKF6 } & \text { Normal } / 100 \% \text { diploid }\end{array}$

OKF6D1/ $\Delta$ p53 Modal number $=47-49, \mathrm{XY}$ : tetraploid chromosome rearrangements:

1. Deletion or translocation of ch 3q.

2. Extra copy or fusion of ch 5 .

3. Extra copy or fusion of ch 7.

4. Deletion or translocation of ch 8.

5. Extra copy of ch 9.

6. Extra copy or fusion of ch 7.

7. Abnormal ch 11q; translocation/fusion (11:23q), possible inverted duplication of 11q. Does not appear to involve locus of cyclin D1 at 11q13.

8. Extra copy of ch 14.

9. Abnormal ch 16: 3p:16q

10. Extra copy of ch 20.

Karyotypic analysis was done at different population doubling times by the University of Pennsylvania Cytogenetics Core Facility on at least ten metaphase spreads. Chromosomal changes displayed occurred in the majority of the spreads. Sporadic changes are not displayed. ch, chromosome. 


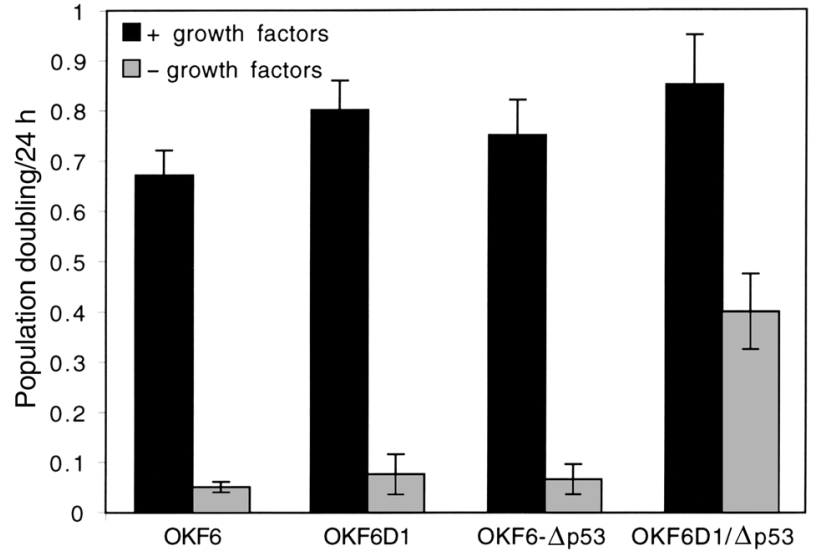

Figure 6

Growth factor dependence of the parental and derived oral keratinocytes. OKF6, OKF6-D1, OKF6- $\Delta$ p53, and OKF6-D1/ $\Delta$ p53 cells were plated at low density either in growth factor-supplemented (+ growth factors) or reduced (- growth factors) medium. Cells were counted 7-9 days later, and growth under these conditions was calculated as PDs per day. Results shown are an average of three experiments.

um. Nonimmortalized oral keratinocytes (OKF6, OKF6-D1, OKF6- $\Delta \mathrm{p} 53$ ) completely retained dependence on growth factors (Figure 6) and did not grow in growth factor-reduced medium. By contrast, the immortalized OKF6-D1/ $\Delta \mathrm{p} 53$ cell strains displayed a proliferative capacity, albeit reduced to $40 \%$ (Figure 6).

To assess whether the immortalized oral keratinocytes might have undergone malignant transformation, we injected the OKF6-D1/ $\Delta \mathrm{p} 53$ cell strains into immunodeficient nude mice. As with OKF6 cells serving as negative controls, no tumors were observed with the OKF6-D $1 / \Delta \mathrm{p} 53$ cell strains after 3 months of observation (Table 2). As positive controls, the human squamous epithelial cancer cell lines SCC15 (oral) and TE 12 (esophageal) readily formed tumors in this assay (Table 2).

\section{Discussion}

One major difference between normal mammalian cells and cancer cells is their proliferative potential. Normal mammalian cells proliferate for a limited number of PDs and then enter a nondividing state known as senescence (3). Cancer cells are able to proliferate indefinitely and are therefore referred to as immortalized. Cellular immortalization is an essential step toward malignant transformation of normal cells. Important genetic changes associated with immortalization are loss of p53 and $\mathrm{pRb}$ functions, and activation of a telomere maintenance mechanism, typically telomerase (7).

That viral oncoproteins such as HPV16 E6 and E7, as well as SV 40 large $T$ antigen, contribute to immortalization already indicates that both $\mathrm{p} 53$ and pRb pathways are involved $(15,16)$. Regardless of the precise mechanism by which the disruption of the p53 and $\mathrm{pRb}$ pathways induces immortalization, their importance is further supported by the well-known observa- tion that these pathways are frequently altered in primary tumors and tumor-derived cell lines $(28,29)$. In this context, cyclin D1 overexpression or loss of p16/INK4A may be viewed as equivalent to loss of $\mathrm{pRb}$ function. Cyclin D1 overexpression is observed in different cancer types, especially of squamous origin, indicating a critical role of the cyclin D1 oncogene in carcinogenesis of this cell type $(19,20)$. Cyclin D1 overexpression is not only a frequent genetic event in the majority of oral cancers, but its expression is correlated with reduced disease-free and overall survival in this cancer (30). The oncogenic role of cyclin D1 was initially established in murine cells as cooperating with the ras, E1A, and neu oncogenes (31-33).

p53 is frequently mutated in oral squamous cell carcinomas. In oral premalignant lesions, expression of p53-positive cells in the suprabasal layers of the epithelium has been seen as an indication of impending malignant development. The prognostic significance of $p 53$ gene mutations in these tumors has been demonstrated (34). That the loss of p53 function is directly associated with escape from senescence was initially demonstrated through the introduction of a dominant-negative p53 gene in fibroblasts (35). Dominant-negative $\mathrm{p} 53$ permits fibroblasts to proliferate for a limited number of PDs, beyond the point at which their normal counterparts become senescent. Evidence that this was attributable to loss of wild-type p53, and not gain of $\mathrm{p} 53$ function, was provided by studies with Li-Fraumeni fibroblasts (36).

Telomerase activity has been associated with many different tumor types. Up to $80 \%$ of primary head and neck squamous cell cancer specimens possessed telomerase activity, and its activity in oral rinses was proposed as a potential molecular marker (11). Several lines of evidence have implicated telomere erosion as the major checkpoint in limiting the proliferative potential of human cells (9). With each cell division, a portion of telomeric DNA is lost. Once the telomeres shorten to a critical length, the signal for the senescence program is activated. This telomere signal that activates the program of senescence could operate through the $\mathrm{pRb}$ and $\mathrm{p} 53$ pathways. Viral oncoproteins HPV E6 and E7 reverse the senescence program by inducing telomerase activation and targeting p53 and $\mathrm{pRb}$ (37). By contrast, expression of the catalytic subunit of telomerase independently was implicated

\section{Table 2}

Formation of tumors in immunodeficient nude mice

$\begin{array}{lc}\text { Cells } & \text { No. of tumors } / \text { no. of injections } \\ \text { OKF6 } & 0 / 8 \\ \text { OKF6D1/ } \Delta \text { p53 } & 0 / 8 \\ \text { TE } 12 & 8 / 8 \\ \text { SCC15 } & 8 / 8\end{array}$

Results are shown for polyclonal populations. Analysis of two individual clones derived from each population gave identical results. $2 \times 10^{6}$ cells were injected in each experiment. Tumor formation was scored as negative after 3 months of monitoring. 
in extension of the replicative life span in a subset of cells without involving pRb and p53 pathways (12). Other cells, including keratinocytes, appear to require at least additional loss of the pRb-mediated cell-cycle control mechanism as a major event toward immortalization $(13,14)$. Recently, it has been demonstrated that the nature of the cell culture conditions might also be an important factor in this cell type-specific process of immortalization (8).

We have demonstrated that OKF6 can extend their limited replicative life span by overexpressing the cyclin $\mathrm{D} 1$ oncogene. Combining this with inactivation of p53 leads to immortalization of the oral keratinocytes. Furthermore, the immortalized epithelial cells (OKF6$\mathrm{D} 1 / \Delta \mathrm{p} 53)$ show reproducibly no increase in telomerase activity. Thus, the immortalization of oral keratinocytes induced by overexpression of cyclin D1 and inactivation of wild-type p53 appears to be independent of telomerase activation. $\mathrm{pRb} / \mathrm{p} 53$ inactivation permitted continuous cell division beyond senescence and progressive telomere shortening. This result does not involve selection of cells with rare genetic alterations, which was confirmed through independent transduction experiments. In contrast to the results presented here, immortalization of epithelial cells using viral oncoproteins HPV E6 and E7 involves E6-dependent activation of telomerase (13).

Expression of SV 40 large T antigen in primary cells leads to inactivation of the pRb and p53 tumor suppressor pathways and allows such cells to grow beyond senescence. Such post-senescent cells lose telomeric sequences corresponding to continued cell division until they reach crisis, when telomeres are critically short and cells experience marked genetic instability (38). Surviving cells maintained telomere length through either activation of telomerase or utilization of ALT thought to involve a recombination based mechanism. Here we show that the combination of cyclin D1 overexpression and p53 inactivation leads to immortalization of oral keratinocytes without activation of telomerase. Telomeres in these immortalized cells are long and heterogeneous, suggesting that such cells maintain telomere length through ALT. Although most immortal cell lines and human tumors are believed to maintain telomere length through activation of telomerase, $10 \%-15 \%$ of tumors (39) and up to $40 \%$ of SV 40 -immortalized cell lines lack detectable telomerase (40). The mechanism of ALT in mammalian cells is not yet understood, whereas in yeast, ALT is accomplished through recombination involving $R A D$ 52 -mediated DNA recombination $(41,42)$.

The fact that telomere length, not telomerase activation, is the biochemical feature most closely associated with immortalization is illustrated by the telomerasenegative immortalized cells $(39,40)$ and is further supported by studies with telomerase dysfunctional mice (43). Although telomere biology may be different in mice, cells from late-generation telomerase-deficient mice are also forced to use alternative mechanisms capa- ble of maintaining telomeres. Studies on mouse embryo fibroblasts (MEFs) from telomerase- and p53-deficient mice demonstrate that p53 mediates the adverse effects of critically short telomeres (44). Coincident with severe telomere shortening and associated genetic instability, p53 is activated, thereby leading to growth arrest and/or apoptosis (45). Progressive telomere shortening in oral keratinocytes may induce the p53 and likely the pRb pathways. High levels of $\mathrm{p} 16$ expression in presenescent OKF6-D1 cells in our study support this notion, as it has been shown that senescence is accompanied by increased p16 expression $(4,46)$. Disruption of p53 function in concert with cyclin D1 overexpression reproducibly enables oral keratinocytes to overcome senescence, thereby leading to immortalization despite maintained $p 16$ levels.

Telomere shortening in cells from telomerase-deficient mice results in loss of telomere function and consequently genetic instability with chromosomal end-toend fusions (47). Cells with short telomeres and intact p53 response may be efficiently eliminated from the culture by apoptosis or growth arrest. Consequently, cells from telomerase-deficient and $\mathrm{p} 53^{-/-}$or $\mathrm{p} 53^{+/-}$ mice display increased chromosomal instability (44, 48). Although these mice may not represent the ideal model for human carcinogenesis owing to the differences in telomere biology $(49,50)$, it is compelling that mice deficient for telomerase and p53, which acquire genetic instability and force cells to use ALT, notably develop squamous epithelial cancers among other tumor types (48). By analogy, disruption of p53 function in combination with cyclin D1 overexpression in human oral keratinocytes induces the same type of genetic instability, including end-to-end fusions and translocations. These chromosomal rearrangements are consistent with the selection of chromosomal changes in cells containing critically short telomeres, which could also have led to the selection of ALT.

Surprisingly, we did not detect widespread cell death or apoptosis characteristic of crisis during the passage of OKF6-D1/ $\Delta \mathrm{p} 53$ cells. Instead, we observed that telomere length shortened in these cells until PD 120. We subsequently observed striking telomere lengthening by PD 160. Although we did not detect evidence for crisis, karyotype analysis of these immortal cells showed clear evidence of end-to-end fusions often seen in cells that have survived crisis. We suspect that the expression of cyclin D1 and dominant-negative p53 permitted the activation of ALT at high frequency in our cultures; however, we cannot yet determine whether the expression of these genes merely created a milieu that fosters the activation of ALT or if it directly induced ALT. Importantly, although ALT cell lines have been derived from cells expressing SV40 large T antigen, the immortalized cells presented here are the first of human epithelial origin to have been generated using genes known to be altered in human cancers. This implies that similar mechanisms may operate during squamous cell carcinogenesis. 
In summary, overexpression of cyclin D1 together with a dominant-negative form of p53 led to the immortalization of oral keratinocytes at high frequency through a telomerase-independent ALT mechanism. This system represents the first model in which ALT was generated using genetic alterations commonly observed in human cancers. We anticipate that this model system will enable us to understand not only the mechanism of ALT activation but also to delineate further the discrete steps that lead to squamous cell malignant transformation.

\section{Acknowledgments}

This work was supported by grants from the NIH (R01DK53377 and P01-DE12467 to A.K. Rustgi), by the Abramson Family Cancer Research Institute (A.K. Rustgi), by grants from Deutsche Krebshilfe (D/96/17197 and 10-1656-Op 1 to O.G. Opitz), by the Howard Hughes Medical Institute Postdoctoral Fellowship (W.C. Hahn), and by a Doris Duke Charitable Foundation Clinical Scientist Development Award (W.C. Hahn). We are grateful to Steven Reeves for the cyclin D1 retroviral expression vector; to Ed Harlow for cyclin D1 and p16 antibodies; and to Hiroshi Nakagawa, Felix Brembeck, and Jun-ichi Okano for discussions. Gita Goessel provided excellent technical assistance. We are also grateful for the advice and support of Robert A. Weinberg.

1. Ness, S.L., et al. 1998. Mouse keratin 4 is necessary for internal epithelial integrity. J. Biol. Chem. 273:23904-23911.

2. Opitz, O.G., Jenkins, T.D., and Rustgi, A.K. 1998. Transcriptional regulation of the differentiation-linked human $\mathrm{K} 4$ promoter is dependent upon esophageal-specific nuclear factors. J. Biol. Chem. 273:23912-23921.

3. Hayflick, L. 1965. The limited in vitro lifespan of human diploid cell strains. Exp. Cell Res. 37:614-621.

4. Loughran, O., et al. 1997. Evidence for the inactivation of multiple replicative lifespan genes in immortal human squamous cell carcinoma keratinocytes. Oncogene. 14:1955-1964.

5. Allsopp, R.C., et al. 1992. Telomere length predicts replicative capacity of human fibroblasts. Proc. Natl. Acad. Sci. USA. 89:10114-10118.

6. Elenbaas, B., et al. 2001. Human breast cancer cells generated by oncogenic transformation of primary mammary epithelial cells. Genes Dev. 15:50-65.

7. Reddel, R.R. 2000. The role of senescence and immortalization in carcinogenesis. Carcinogenesis. 21:477-484.

8. Ramirez, R.D., et al. 2001. Putative telomere-independent mechanisms of replicative aging reflect inadequate growth conditions. Genes Dev. 15:398-403.

9. Harley, C.B., Futcher, A.B., and Greider, C.W. 1990. Telomeres shorten during aging of human fibroblasts. Nature. 345:458-460.

10. Kim, N.W., et al. 1994. Specific association of human telomerase activity with immortal cells and cancer. Science. 266:2011-2015.

11. Califano, J., et al. 1996. Detection of telomerase activity in oral rinses from head and neck squamous cell carcinoma patients. Cancer Res. 56:5720-5722.

12. Bodnar, A.G., et al. 1998. Extension of life-span by introduction of telomerase into normal human cells. Science. 279:349-352.

13. Kiyono, T., et al. 1998. Both Rb/p16INK4a inactivation and telomerase activity are required to immortalize human epithelial cells. Nature. 396:84-88.

14. Dickson, M.A., et al. 2000. Human keratinocytes that express hTERT and also bypass a p16(INK4a)- enforced mechanism that limits life span become immortal yet retain normal growth and differentiation characteristics. Mol. Cell. Biol. 20:1436-1447.

15. Wright, W.E., Pereira-Smith, O.M., and Shay, J.W. 1989. Reversible cellular senescence: implications for immortalization of normal human diploid fibroblasts. Mol. Cell. Biol. 9:3088-3092.

16. Hawley-Nelson, P., Vousden, K.H., Hubbert, N.L., Lowy, D.R., and Schiller, J.T. 1989. HPV16 E6 and E7 proteins cooperate to immortalize human foreskin keratinocytes. EMBO J. 8:3905-3910.

17. Bryan, T.M., and Reddel, R.R. 1994. SV40-induced immortalization of human cells. Crit. Rev. Oncog. 5:331-357.

18. Hahn, W.C., et al. 1999. Creation of human tumour cells with defined genetic elements. Nature. 400:464-468.

19. Bartkova, J., et al. 1995. Abnormal patterns of D-type cyclin expression and G1 regulation in human head and neck cancer. Cancer Res. 55:949-956.
20. Nakagawa, H., et al. 1995. Human cyclin D1 oncogene and esophageal squamous cell carcinoma. Cancer. 76:541-549.

21. Nakagawa, H., et al. 1997. The targeting of the cyclin D1 oncogene by an Epstein-Barr virus promoter in transgenic mice causes dysplasia in the tongue, esophagus and forestomach. Oncogene. 14:1185-1190.

22. Wong, D.T., Todd, R., Tsuji, T., and Donoff, R.B. 1996. Molecular biology of human oral cancer. Crit. Rev. Oral Biol. Med. 7:319-328.

23. Lindberg, K., and Rheinwald, J.G. 1990. Three distinct keratinocyte subtypes identified in human oral epithelium by their patterns of keratin expression in culture and in xenografts. Differentiation. 45:230-241.

24. Hiyama, H., Iavarone, A., LaBaer, J., and Reeves, S.A. 1997. Regulated ectopic expression of cyclin D1 induces transcriptional activation of the cdk inhibitor p21 gene without altering cell cycle progression. Oncogene. 14:2533-2542.

25. Opitz, O.G., and Rustgi, A.K. 2000. Interaction between Sp1 and cell cycle regulatory proteins is important in transactivation of a differentiation-related gene. Cancer Res. 60:2825-2830.

26. Thompson, D.A., et al. 1997. The human papillomavirus-16 E6 oncoprotein decreases the vigilance of mitotic checkpoints. Oncogene. 15:3025-3035.

27. Hahn, W.C., et al. 1999. Inhibition of telomerase limits the growth of human cancer cells. Nat. Med. 5:1164-1170.

28. Harris, C.C. 1993. p53: at the crossroads of molecular carcinogenesis and risk assessment. Science. 262:1980-1981.

29. Weinberg, R.A. 1995. The retinoblastoma protein and cell cycle control. Cell. 81:323-330.

30. Bova, R.J., et al. 1999. Cyclin D1 and p16INK4A expression predict reduced survival in carcinoma of the anterior tongue. Clin. Cancer Res. 5:2810-2819.

31. Hinds, P.W., Dowdy, S.F., Eaton, E.N., Arnold, A., and Weinberg, R.A. 1994. Function of a human cyclin gene as an oncogene. Proc. Natl. Acad. Sci. USA. 91:709-713.

32. Lee, R.J., et al. 2000. Cyclin D1 is required for transformation by activated Neu and is induced through an E2F-dependent signaling pathway. Mol. Cell. Biol. 20:672-683.

33. Lovec, H., Sewing, A., Lucibello, F.C., Muller, R., and Moroy, T. 1994. Oncogenic activity of cyclin D1 revealed through cooperation with Ha-ras: link between cell cycle control and malignant transformation. Oncogene. 9:323-326.

34. Nylander, K., Dabelsteen, E., and Hall, P.A. 2000. The p53 molecule and its prognostic role in squamous cell carcinomas of the head and neck. J. Oral Pathol. Med. 29:413-425.

35. Bond, J.A., Wyllie, F.S., and Wynford-Thomas, D. 1994. Escape from senescence in human diploid fibroblasts induced directly by mutant $\mathrm{p} 53$. Oncogene. 9:1885-1889.

36. Rogan, E.M., et al. 1995. Alterations in p53 and p16INK4 expression and telomere length during spontaneous immortalization of Li-Fraumeni syndrome fibroblasts. Mol. Cell. Biol. 15:4745-4753.

37. Klingelhutz, A.J., Foster, S.A., and McDougall,J.K. 1996. Telomerase activation by the E6 gene product of human papillomavirus type 16. Nature. 380:79-82.

38. Counter, C.M., et al. 1992. Telomere shortening associated with chromosome instability is arrested in immortal cells which express telomerase activity. EMBOJ. 11:1921-1929.

39. Bryan, T.M., Englezou, A., Dalla-Pozza, L., Dunham, M.A., and Reddel, R.R. 1997. Evidence for an alternative mechanism for maintaining telomere length in human tumors and tumor-derived cell lines. Nat Med. 3:1271-1274.

40. Bryan, T.M., and Reddel, R.R. 1997. Telomere dynamics and telomerase activity in in vitro immortalised human cells. Eur. J. Cancer. 33:767-773.

41. Lundblad, V., and Blackburn, E.H. 1993. An alternative pathway for yeast telomere maintenance rescues est1- senescence. Cell. 73:347-360.

42. McEachern, M.J., and Blackburn, E.H. 1996. Cap-prevented recombination between terminal telomeric repeat arrays (telomere CPR) maintains telomeres in Kluyveromyces lactis lacking telomerase. Genes Dev. 10:1822-1834.

43. Blasco, M.A., et al. 1997. Telomere shortening and tumor formation by mouse cells lacking telomerase RNA. Cell. 91:25-34.

44. Chin, L., et al. 1999. p53 deficiency rescues the adverse effects of telomere loss and cooperates with telomere dysfunction to accelerate carcinogenesis. Cell. 97:527-538.

45. Karlseder, J., Broccoli, D., Dai, Y., Hardy, S., and de Lange, T. 1999. p53- and ATM-dependent apoptosis induced by telomeres lacking TRF2. Science. 283:1321-1325.

46. Dai, C.Y., and Enders, G.H. 2000. p16 INK4a can initiate an autonomous senescence program. Oncogene. 19:1613-1622.

47. Hande, M.P., Samper, E., Lansdorp, P., and Blasco, M.A. 1999. Telomere length dynamics and chromosomal instability in cells derived from telomerase null mice. J. Cell. Biol. 144:589-601.

48. Artandi, S.E., et al. 2000. Telomere dysfunction promotes non-reciprocal translocations and epithelial cancers in mice. Nature. 406:641-645.

49. Wright, W.E., and Shay, J.W. 2000. Telomere dynamics in cancer progression and prevention: fundamental differences in human and mouse telomere biology. Nat. Med. 6:849-851.

50. Artandi, S.E., and DePinho, R.A. 2000. Mice without telomerase: what can they teach us about human cancer? Nat. Med. 6:852-855. 